\title{
Clinical presentation of scrub typhus during a major outbreak in central Nepal
}

\author{
Arun Sedhain ${ }^{1}$, Gandhi R Bhattarai ${ }^{2}$ \\ ${ }^{1}$ Associate Professor, Department of Medicine, Nephrology unit, Chitwan Medical College, Bharatpur, Chitwan, Nepal, \\ ${ }^{2}$ Director, Health Services Research, United Health Group, OptumInsight, CT, USA.
}

Background: Scrub typhus, an emerging rickettsial disease caused by the organism Orientiatsutsugamushi, is associated with multi-organ involvement. We prospectively studied the clinical manifestations of the disease during a major outbreak in central part of Nepal. Aims and Objective: This study was carried out with an aim to analyze the clinical presentations, laboratory parameters, complications and outcomes of scrub typhus. Materials and Methods: A prospective observational study was conducted in the Department of Medicine in a tertiary teaching hospital. A total of 1398 patients admitted with acute febrile illness were subjected for Scrub Typhus Detect ${ }^{\mathrm{TM}}$ IgM ELISA test, among which 502 (35.90\%) patients tested positive and were included in the study. Acute kidney injury was defined according to KDIGO guideline. Statistical analysis was done with SAS University Studio package using t-test for continuous variables and chi-square test for categorical variables. Results: Mean age of the patients was $30.37 \pm 18.81$ years with $26.29 \%$ in the pediatric age group ( $<14$ years). Females comprised of $55.98 \%$ of the patients. Majority $(97.98 \%)$ of the patients were seen between July to November. Clinical presentations in the descending order of frequency were anorexia (55.18\%), headache (53.39\%), lymphadenopathy (15.73\%), jaundice (13.35) and eschar $(6.57 \%)$. Mean scrub typhus IgM ELISA value was 2.17. Leukocytosis was seen in $5.78 \%$ and thrombocytopenia in $66.73 \%$ of patients. Transaminitis was found in $80.68 \%$ of patients. Urinary abnormalities were recorded in $42.3 \%$ of patients. Acute kidney injury (AKI) was seen in $35.8 \%$ of patients followed by acute respiratory distress syndrome $(24.1 \%)$, pneumonia $(22.1 \%)$, shock $(14.74 \%)$, neurologic manifestation $(6.37 \%)$ and cardiac manifestations $(4.38 \%)$. ICU admission was required for $18.73 \%$ of patients and $8.57 \%$ required ventilator support. Mortality rate was $1.79 \%$. Conclusion: Scrub typhus, being an emerging vector borne infectious disease in Nepalese context, is associated with multiple organ involvement.

Key words: Acute febrile illness, Acute kidney injury (AKI), Hematuria, Albuminuria

\section{INTRODUCTION}

Scrub typhus is a rickettsial disease caused by the organism Orientia tsutsugamushi and transmitted through the bite of larval forms (chiggers) of trombiculid mites. ${ }^{1}$ It presents as either a non-specific febrile illness with constitutional symptoms such as fever, rash, myalgias and headache or with multi-organ dysfunctions involving organs such as kidney, liver, lungs, central nervous system or with circulatory collapse. $^{2}$
Access this article online

Website:

http://nepjol.info/index.php/AJMS

DOI: 10.3126/ajms.v8i4.17163

E-ISSN: 2091-0576

P-ISSN: 2467-9100
Scrub typhus as a cause of acute febrile illness in Nepal was first reported in 1981. ${ }^{3}$ Hospital based study conducted in 2004 in central Nepal found scrub typhus as a cause of acute febrile illness in $3 \%$ of patients. ${ }^{4}$ Since then the disease has been considered to be endemic in different parts of the country, though it did not cause significant problems. After a 7.5 magnitude earthquake in April 2015, major outbreak of scrub typhus was noted in different districts of central Nepal. So, this study was carried out with an aim to analyze the clinical 
presentations, laboratory parameters, complications and outcomes of scrub typhus.

\section{MATERIALS AND METHODS}

A prospective analytical study was conducted from April to December 2016 in the Department of Medicine at Chitwan Medical College Teaching Hospital, a tertiary care institution located in Central Nepal. Approval from the ethical committee and informed written consent from the patients were taken prior to the study. Patients admitted with acute febrile illness (AFI) who were tested positive for Scrub Typhus Detect ${ }^{\mathrm{TM}}$ IgM ELISA test (titer equal or more than 0.5), were included in the study. Patients who concomitantly had other infections like dengue, laptopirosis, typhoid fever, brucella or malaria were excluded from the study.

Demographic, clinical and laboratory variables were recorded in all patients. All patients had a detailed clinical history and examination, a standard set of investigations including complete blood counts, liver function tests, serum urea, creatinine, electrolytes, chest radiograph and ultrasonography of abdomen and pelvis. Leukocytosis was defined as white blood cells [WBC] $>12000$ cells $/ \mathrm{mm} 3$, anemia as hemoglobin $<10$ gram $/ \mathrm{dL}$, thrombocytopenia as platelets $<1.5$ lakhs $/ \mathrm{mm} 3$, elevated bilirubin as total bilirubin $>3 \mathrm{mg} / \mathrm{dL}$ and elevated aspartate aminotransferase and alanine aminotransferase as four times the normal. Urinary abnormalities were defined by dipstick proteinuria, pyuria, hematuria and casts as seen on urine microscopy and Acute Kidney Injury (AKI) was defined as per kidney disease improving global outcome (KDIGO) guideline. ${ }^{5}$ Patient characteristics were summarized using mean and frequency distributions. Data for continuous variables were expressed as mean \pm standard deviation using t-test for continuous variables and chi-square test for categorical variables. Analysis of Variation (ANOVA) tests were done to test the differences of continuous variables across multiple groups. Descriptive and inferential statistical analysis were done by using SAS University Studio package.

\section{RESULTS}

Out of 1398 patients with AFI subjected for Scrub Typhus Detect ${ }^{\mathrm{TM}}$ IgM ELISA test, 502 (35.90\%) patients were tested positive. Mean age of the patients was $30.37 \pm 18.81$ years with an age range between 1 to 79 years (Table 1). Almost $26 \%$ of the patients were in the pediatric age group ( $<14$ years). Females comprised of $55.98 \%$ of the patients with female to male ratio of 1.26:1.
Majority of the patients were from the plain area $(72 \%)$ of the country and most of them were from the rural $(32 \%)$ and semi-urban (24\%) areas (Figure 1).

The first case of scrub typhus during this outbreak was seen in April and majority (97.98\%) of the patients were seen between July to November with highest number of patients seen in October (Figure 2).

All 100\% patients included in the study had fever. Other clinical presentations in the descending order of frequency were anorexia, headache, lymphadenopathy, jaundice and eschar. Urinary abnormalities in $42.3 \%$ of patients (Table 2 ).

Most common complication seen was acute kidney injury (AKI), of which $66 \%$ had stage $1,33 \%$ had stage 2 and $1 \%$ had stage 3 AKI. Hemodialysis was required in 1.79\% of patients. Other complications in descending order were

\begin{tabular}{lc}
\multicolumn{2}{l}{ Table 1: Baseline clinical characteristics } \\
\hline Variables & Mean \pm SD \\
\hline Age (years) & $30.37 \pm 18.81$ \\
IgM ELISA value & $2.17 \pm 1.70$ \\
Days in the hospital & $5.39 \pm 2.32$ \\
Days in ICU & $1.07 \pm 2.35$ \\
Duration of fever (days) & $5.57 \pm 1.22$ \\
\hline
\end{tabular}

\begin{tabular}{lc} 
Table 2: Clinical presentations of scrub typhus \\
\hline Clinical parameters & Number (\%) \\
\hline Anorexia & $277(55.18)$ \\
Headache & $268(53.39)$ \\
Lymphadenopathy & $79(15.73)$ \\
Jaundice & $67(13.35)$ \\
Eschar & $33(6.57)$ \\
\hline
\end{tabular}

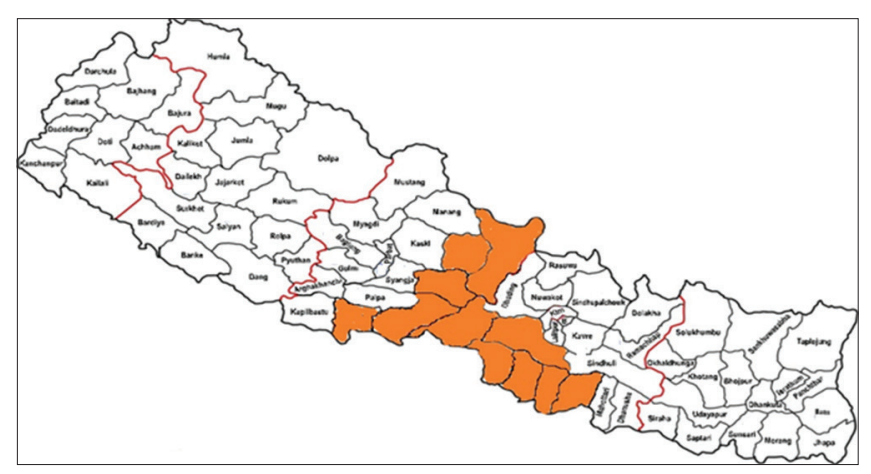

Figure 1: Geographic distribution of the patients

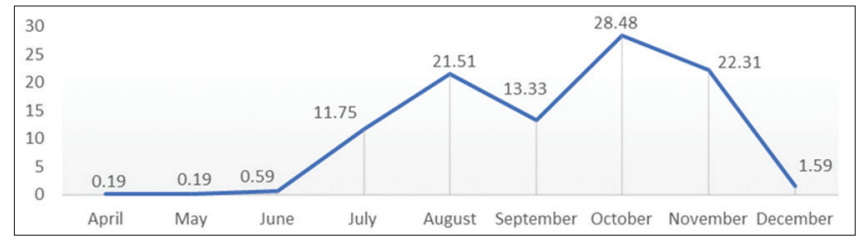

Figure 2: Month-wise distribution of patients 
acute respiratory distress syndrome (ARDS), pneumonia, shock, neurologic manifestation and cardiac manifestations. Admission to ICU was required for $18.73 \%$ of patients with mean duration of ICU stay of 1.06 days. Mechanical ventilation was needed for $8.57 \%$ of patients. Nine patients died with a mortality rate of $1.79 \%$ (Table 3 ).

Leukocytosis (WBC count $>11,000 / \mathrm{mm} 3$ ) was seen in $5.78 \%$ and thrombocytopenia in $66.73 \%$ of patients. Transaminitis was seen in $80.68 \%$ of patients with majority having raised AST than ALT (90.63 versus $86.65 \%$; $\mathrm{p}=0.531$ ). Mean scrub typhus IgM ELISA value was 2.17 (Table 4).

\section{DISCUSSION}

This is the first report to comprehensively document the clinical presentation, laboratory investigation, pattern of renal involvement and outcome in a cohort of patients of scrub typhus in Nepal. We have found that scrub typhus was responsible for about $36 \%$ of patients presenting with unexplained febrile illness.

Scrub typhus is a vector borne disease caused by Orientia tsutsugamushi, an obligate intracellular gram negative bacteria and transmitted by the bite of an infected Trombiculid mite larva. ${ }^{6}$ The disease was first described from Japan in 1899 and in the initial days the overall mortality varied from $7 \%$ to $9 \% .^{7}$ In the current days scrub typhus is endemic to a part of the world known as the "tsutsugamushi triangle", which extends from northern Japan and far-eastern Russia in the north, to northern

\begin{tabular}{lcc}
\multicolumn{3}{l}{ Table 3: Complications in scrub typhus } \\
\hline Complications & Number & Percentage (mean \pm SD) \\
\hline AKI & 180 & $35.82 \pm 23.72$ \\
ARDS & 120 & $24.10 \pm 42.81$ \\
Pneumonia & 110 & $22.1 \pm 41.54$ \\
ICU admission & 94 & $18.73 \pm 58.24$ \\
Shock & 74 & $14.74 \pm 35.49$ \\
Ventilatory support & 43 & $8.57 \pm 28.01$ \\
Neurologic manifestation & 32 & $6.37 \pm 24.45$ \\
Cardiac manifestation & 22 & $4.38 \pm 20.49$ \\
Death & 9 & $1.79 \pm 13.28$ \\
\hline
\end{tabular}

\section{Table 4: Baseline laboratory parameters}

\begin{tabular}{lc}
\hline Parameter & Value (mean \pm SD) \\
\hline TLC (cumm) & $7327.63 \pm 2364.82$ \\
$\mathrm{Hb}(\mathrm{gm} \%)$ & $10.26 \pm 0.98$ \\
$\mathrm{PLC}(\mathrm{cumm})$ & $116689.86 \pm 58172.49$ \\
$\mathrm{AST}(\mathrm{U} / \mathrm{L})$ & $72.48 \pm 53.09$ \\
$\mathrm{ALT}(\mathrm{U} / \mathrm{L})$ & $64.40 \pm 44.93$ \\
Urea $(\mathrm{mg} / \mathrm{dL})$ & $30.09 \pm 12.05$ \\
Creat $(\mathrm{mg} / \mathrm{dL})$ & $1.36 \pm 0.69$ \\
Scrub ELISA & $2.17 \pm 1.70$ \\
\hline TLC: Total leucocyte count, PLC: Patelet count, AST: Aspartate aminotransferase, \\
ALT: Alanine aminotransferase
\end{tabular}

Australia in the south and to Pakistan in the west. ${ }^{8}$ In Nepal, first study to detect scrub typhus was done in 1981 in eastern part of the country where $10 \%$ of study population were found to have antibodies to scrub-typhus rickettsiae, indicating that the infection occurred widely in eastern Nepal. ${ }^{3}$ In another study, up to $3 \%$ of in-hospital patients in central Nepal were found to be infected with the scrub typhus. ${ }^{4}$ However, till date there had not been such a large outbreak and clinical study of scrub typhus in the country. Current outbreak of scrub typhus in Nepal was seen from those areas which were affected by the 7.8 magnitude earthquake in April 2015. ${ }^{9}$ These outbreaks were thought to be due to people and rodents living in close proximity in temporary shelters after the earthquake. ${ }^{10,11}$ Majorities of the patients included in our study were from the earthquake affected areas (Figure 1).

The bite of the mite leaves a characteristic black eschar. The adult mites have a four-staged lifecycle viz. egg, larva, nymph and adult. The larval stage, also known as chigger, is the only stage that can transmit the disease to humans and other vertebrates. ${ }^{6}$ The incubation period of scrub typhus in human is around 10-12 days and the clinical manifestation varies from mild febrile illness to severely fatal multiorgan dysfunction syndrome. Apart from the non-specific generalized clinical features like fever, malaise, anorexia, headache, lymphadenopathy and cough, ${ }^{12}$ the significant findings of scrub typhus is eschar formation at the site of bite, the prevalence of which is variable ranging from $7 \%$ to $80 \%{ }^{13,14}$ However, eschar is rarely seen in south East Asia and Indian subcontinent. ${ }^{15,16}$ Lymphadenopathy usually painful, is a common finding in scrub typhus reported in 13 to $18 \%$ of patients. ${ }^{17-19}$ In this study, all patients had fever followed by anorexia, headache, jaundice and lymphadenopathy. Eschar formation was seen only in $8 \%$ of the patients.

Diagnosis of scrub typhus requires a high degree of clinical suspicion, which is confirmed serologically by different laboratory investigations. ${ }^{20}$ The cheapest test currently available and used extensively in Indian subcontinent is Weil- Felix test which is highly specific, but lacks sensitivity. ${ }^{21,22}$ Immunoglobulin M (IgM) enzyme-linked immunosorbent assay (ELISA) using the $56 \mathrm{kDa}$ antigen has been widely used, which is easy to perform, gives quick results and has sensitivity of 84 to $86 \%$ and a specificity of $98 \%{ }^{23,24}$ and is well validated. ${ }^{25}$ No current diagnostic test is sufficiently practical for use by physicians working in rural areas. A new dipstick test using a dot blot immunoassay format appears to be the best currently available test for diagnosing scrub typhus in rural areas where this disease predominates. ${ }^{26}$

Reported severe clinical manifestations or complications of scrub typhus include AKI, meningoencephalitis, 
myocarditis, pneumonia, ARDS, AKI, GI bleeding, septic shock, and multiple organ failures which may be potentially fatal if there is a delay in diagnosis and treatment. ${ }^{27-33}$ Acute kidney injury (AKI) and renal involvement in scrub typhus is believed to be multifactorial in origin. Overall, renal involvement is considered to be a part of multi-organ dysfunction syndrome in patients with severe disease. In this study, $35.8 \%$ of the patients developed AKI, which is consistent with findings in other studies where its incidence has been reported ranging from 10 to $60 \%{ }^{32-36}$ Most of the patients had stage 1 and 2 AKI comprising of $66 \%$ and $33 \%$ respectively and only $1 \%$ of patients had stage $3 \mathrm{AKI}$.

Urinary abnormalities have been reported in 50\% to $80 \%$ of patients with scrub typhus. ${ }^{37}$ Attur et al in their study from south India reported urinary abnormalities in all patients with $\mathrm{AKI} \cdot{ }^{32}$ Urinary abnormalities were seen in $52.94 \%$ of patients with proteinuria being the most common presentation $(23.71 \%)$ followed by hematuria $(17.73 \%)$ pyuria $(5 \%)$ gross hematuria $(4.5 \%)$ and dipstick positive glycosuria $(2 \%)$. The incidence of these urinary abnormalities suggest intrinsic renal involvement being the most likely explanation.

Mortality was $1.79 \%$, which was significantly higher in the AKI than in the non-AKI group (2.96\% versus $1.0 \%$; $\mathrm{p}=0.005)$. Higher rate of mortality in AKI patients could be the reflection of underlying severity of disease. Our finding is comparable to other studies, which ranged from $0.79 \%$ to $12 \% .{ }^{38,39}$ Vivekanandan et al on their study from south India reported the mortality of $12.2 \%$. Lower mortality in our study was probably due to early diagnosis and initiation of treatment. Delayed presentation, acute respiratory distress syndrome and shock have been shown to be the determinants of mortality in previous studies.

There are certain limitations to our study. This was a single center study done in central Nepal and might not reflect the national scenario as the outbreak of scrub typhus had been reported from other areas of the country as well. Secondly, the diagnosis of scrub typhus was made based on the ELISA test and we excluded patients with equivocal results (ELISA titer between 0.2 and 0.5). Although the ELISA method has a satisfactory sensitivity and specificity, nucleic acid amplification tests would bring better results.

\section{CONCLUSION}

Scrub typhus is an important cause of febrile illness with multisystem involvement in Nepalese context. If clinicians do not consider a possibility of Rickettsial diseases in a patient with fever, work up for AFI may become difficult and expensive. So, we suggest that scrub typhus should be part of the differential diagnosis in patients with acute febrile illness.

\section{REFERENCES}

1. Mahajan SK. Scrub typhus. J Assoc Physicians India 2005;53:954-958.

2. Chogle AR. Diagnosis and treatment of scrub typhus - the 3 . Indian scenario. J Assoc Physicians India 2010;58:11-12.

3. Brown GW, Shirai A, Gan E and Bernthal P. Antibodies to typhus in Eastern Nepal. Trans R Soc Trop Med Hyg 1981;75(4):586-587.

4. Murdoch DR, Woods CW, Zimmerman MD, Dull PM, Belbase RH, Keenan AJ, et al. The etiology of febrile illness in adults presenting to Patan Hospital in Kathmandu Nepal. Am J Trop Med Hyg 2004;70:670-675.

5. KDIGO clinical practice guideline for acute kidney injury, section 2: AKI definition. Kidney Int Supple 2012;2:19-22.

6. Ramasubramanian $V$ and Senthur Nambi P. Scrub typhus. In: Medicine Update. India: Association of Physicians of India. 2013:19-22.

7. Groves MG, Harrington KS. Scrub typhus. In: Beran GW (Ed). Handbook of Zoonoses, $2^{\text {nd }}$ edition. Florida: CRC Press; 1994. 663-668.

8. McCrumb FR, Stockard JL, Robinson CR, Turner LH, Levis DG, Maisey CW, et al. Leptospirosis in Malaya. I. Sporadic cases among military and civilian personnel. Am J Trop Med Hyg 1957;6(2):238-256.

9. The Kathmandu Post. Rats causing scrub typhus: WHO team. The Kathmandu Post (Kathmandu), Oct 7, 2015. http:// kathmandupost. ekantipur.com/news/2015-10-07/rats-causingscrub-typhus-who-team. html (accessed June 24, 2016).

10. Nayak N. Scrub Typhus in Nepal. Nepal J Epidemiol 2016;6(2);563-564.

11. Basnyat B. Typhoid versus typhus fever in post-earthquake Nepal. The Lancet 2016;4:e516-e517.

12. Jeong YJ, Kim S, Wook YD, Lee JW, Kim KI and Lee SH. Scrub typhus: clinical, pathologic, and imaging findings. Radiographics 2007;27:161-172.

13. Kim DM, Won KJ, Park CY, Yu KD, Kim HS, Yang TY, et al. Distribution of eschars on the body of scrub typhus patients: a prospective study. Am J Trop Med Hyg 2007; 76: 806-809.

14. Sirisanthana V, Puthanakit T and Sirisanthana T. Epidemiologic, clinical and laboratory features of scrub typhus in thirty Thai children. Pediatr Infect Dis J 2003; 22: 341-345.

15. Vivekanandan M, Mani A, Priya YS, Singh AP, Jayakumar S and Purty S. Outbreak of scrub typhus in Pondicherry. J Assoc Physicians India 2010; 58: 24-28.

16. Sharma A, Mahajan S, Gupta ML, Kanga A and Sharma V. Investigation of an outbreak of scrub typhus in the himalayan region of India. Jpn J Infect Dis 2005; 58: 208-210.

17. Narvencar KPS, Rodrigues S, Nevrekar RP, Dias L, Dias A, Vaz, et al. Scrub typhus in patients reporting with acute febrile illness at a tertiary health care institution in Goa. Indian J Med Res 2012;136:1020-1024.

18. Su TH, Liu CJ, Chen DS and Kao JH. Milder clinical manifestation of scrub typhus in Kinmen, Taiwan. J Formos Med Assoc 2013;112:201-207.

19. Zhang M, Zhao ZT, Wang XJ, Li Z, Ding L and Ding SJ. Scrub typhus: surveillance, clinical profile and diagnostic issues in Shandong, China. Am J Trop Med Hyg 2012;87:1099-1104.

20. Mandell GL, Douglas JR and Bennett JE. Principles and practice 
of infectious disease. $7^{\text {th }}$ ed. Philadelphia: Churchill Livingstone; 2010;2529-2530.

21. Batra HV. Spotted fevers and typhus fever in Tamil Nadu. Indian J Med Res 2007;126:101-103.

22. Mahajan SK, Kashyap R, Kanga A, Sharma V, Prasher BS and Pal LS. Relevance of Weil -Felix test in diagnosis of scrub typhus in India. J Asssoc Physicians India 2006; 54: 619-621.

23. Prakash JA, Abraham OC and Mathai E. Evaluation of tests for serological diagnosis of scrub typhus. Trop Doct 2006;36(4):212-213.

24. Blacksell SD, Tanganuchitcharnchai A, Nawtaisong P, et al. Diagnostic Accuracy of the InBios Scrub Typhus Detect EnzymeLinked Immunoassay for the Detection of IgM Antibodies in Northern Thailand. Rosenberg HF, ed. Clinical and Vaccine Immunology : CVI. 2016;23(2):148-154.

25. Varghese GM, Abraham OC, Mathai D, Thomas K, Aaron R, Kavitha ML, et al. Scrub typhus among hospitalised patients with febrile illness in South India: magnitude and clinical predictors. J Infect 2006; 52:56-60.

26. Watt. G. Scrub typhus In Oxford Textbook of Medicine. Warrel DA, Cox TM, Firth JD et al (Edi) $4^{\text {th }}$ Edi. Oxford. Oxford University Press 2003;1:629-631.

27. Chen $\mathrm{PH}$, Hung $\mathrm{KH}$, Cheng SJ and Hsu KN. Scrub typhus associated acute disseminated encephalitis. Acta Neurol Taiwan 2006;15:251-254

28. Aronoff DM and Watt $\mathrm{G}$. Prevalence of relative bradycardia in Orientia tsutsugamushi infection. Am J Trop Med Hyg 2003;68(4):477-479.

29. Raoult D. Orientia tsutsugamushi (Scrub Typhus). In: Mandell GL, Bennett JE, Dolin R, editors. Mandell: Mandell, Douglas, and Bennett's Principles and Practice of Infectious Diseases. Seventh ed: Churchill Livingstone, An Imprint of Elsevier. 2009;2529-30.

30. Reller ME, Dumler JS. Scrub Typhus (Orientia tsutsugamushi). In: Kliegman RM, Stanton BF, GemellI JWS, Schor NF, Behrman
RE, editors. Kliegman: Nelson Textbook of Pediatrics. Nineteenth ed: Saunders, An Imprint of Elsevier. 2011;1045-1046.

31. Wang CC, Liu SF, Liu JW, Chung YH, Su MC and Lin MC. Acute respiratory distress syndrome in scrub typhus. Am J Trop Med Hyg 2007;76(6):1148-1152.

32. Attur RP, Kuppasamy S, Bairy M, Nagaraju SP, Pammidi NR, Kamath V, et al. Acute kidney injury in scrub typhus. Clinical and Experimental Nephrology 2013;17(5);725-729.

33. Kim DM, Kang DW, Kim JO, Chung JH, Kim HL, Park CY, et al. Acute renal failure due to acute tubular necrosis caused by direct invasion of Orientia tsutsugamushi. J Clin Microbiol 2008; 46: 1548-1550.

34. Kumar M, Krishnamurthy S, Delhikumar CG, Narayanan P, Biswal N, et al. Scrub typhus in children at a tertiary hospital in southern India: clinical profile and complications. J Infect Public Health 2012;5:82-88.

35. Basu G, Chrispal A, Boorugu H, Gopinath KG, Chandy S, et al. Acute kidney injury in tropical acute febrile illness in a tertiary care centre-RIFLE criteria validation. Nephrol Dial Transplant 2011;26:524-531.

36. Vikrant S, Dheer SK, Parashar A, Gupta D, Thakur S, et al. Scrub typhus associated acute kidney injury-a study from a tertiary care hospital from western Himalayan state of India. Renal Failure 2013;1-6.

37. Kumar V, Kumar V, Yadav AK, lyengar S, Bhalla A, Sharma N, et al. Scrub typhus is an under-recognized cause of acute febrile illness with acute kidney injury in India. PLoS Negl Trop Dis 2014;8:e2605

38. Chrispal A, Boorugu H, Gopinath KG, Prakash JA, Chandy S, Abraham OC, et al. Scrub typhus: An unrecognized threat in South India-clinical profile and predictors of mortality. Trop Doct 2010;40:129-133.

39. Vivekanandan M, Mani A, Priya YS, Singh AP, Jayakumar S and Purty S. Out Break of Scrub Typhus in Pondicherry. J Assoc Physician India 2009; 57: 802-806.

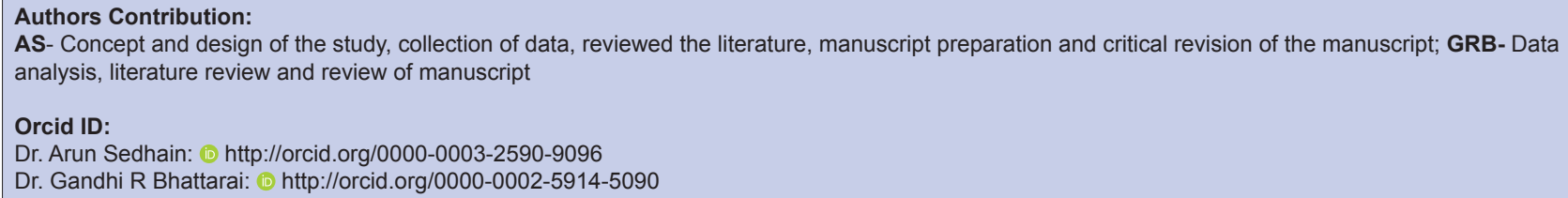

